\section{AB0165 THE EUROPEAN REGISTRY ON OBSTETRIC ANTIPHOSPHOLIPID SYNDROME (EUROAPS): A SURVEY OF 1100 CONSECUTIVE CASES}

Enrique Esteve-Valverde ${ }^{1}$, Jaume Alijotas-Reig ${ }^{2}$, Raquel Ferrer-Oliveras ${ }^{3}$ Luis Sáez-Comet ${ }^{4}$, Cristina Belizna ${ }^{5}$, Amelia Ruffatti $^{6}$, Angela Tincani ${ }^{7}$, Sara Decarolis ${ }^{8}$, Omar Latino ${ }^{9}$, Ricard Cervera ${ }^{10}$, on behalf of EUROAPS study group. ${ }^{1}$ Internal Medicine Department, Althaia Healthcare University Network of Manresa, Systemic Autoimmune Disease Unit, Manresa, Barcelona, Spain., Barcelona, Spain; ${ }^{2}$ Systemic Autoimmune Disease Unit, Department of Internal Medicine, Vall d'Hebron University Hospital, Department of Medicine, Universitat Autonoma, Barcelona, Barcelona, Spain; ${ }^{3}$ Obstetrics and Gynaecology Department, High Risk Unit, Vall d'Hebron University Hospital, Universitat Autonoma Barcelona, Barcelona, Spain; ${ }^{4}$ Internal Medicine Department, Miguel Servet University Hospital, Zaragoza, Spain, ${ }^{5}$ Vascular and Coagulation Department, University Hospital Angers, Angers, France; ${ }^{6}$ Rheumatology Unit, Department of Clinical and Experimental Medicine Azienda Ospedaliera, University of Padua, Padova, Italy; ${ }^{7}$ U.O. Rheumatology and Clinical Immunology, ASST Spedali Civili of Brescia, Brescia, Italy; ${ }^{8}$ Department of Obstetrics, Gynaecology and Pediatrics, F.Policlinico Gemelli IRCCS, Università Cattolica del S.Cuore, Rome, Italy; ${ }^{9}$ Autoimmune, Thrombophilic Diseases and Pregnancy Division,Dr. Carlos G Durand Hospital, Buenos Aires, Argentina; ${ }^{10}$ Systemic Autoimmune Diseases Service, Hospital Clinic, Universitat de Barcelona, Barcelona, Spain

Background: The obstetric antiphospholipid syndrome (OAPS) is an autoimmune disease defined by the presence of obstetric complications related to antiphospholipid antibodies. EUROAPS project is the biggest published European registry on obstetric antiphospholipid syndrome and it is ongoing.

Objectives: To analyse the clinical features, laboratory data and foetal-maternal outcomes, and follow them up on a cohort of 1100 women with obstetric antiphospholipid syndrome (OAPS)

Methods: Thirty hospitals throughout Europe have collaborated to carry out this registry. Cases with obstetric complaints related to antiphospholipid antibodies (aPL) who tested positive for aPL at least twice were included prospectively and retrospectively. The eight-year survey results are reported.

Results: 1100 women with 3653 episodes were included of which 2553 were historical and 1100 were latest episodes. All cases fulfilled the Sydney classification criteria. According to the laboratory categories: $29.2 \%$ were in category I,35.7\% in Ila,22.4\% in Ilb and $12.7 \%$ ) in Ilc. Miscarriages appeared in $38.6 \%$ cases. Early preeclampsia and early foetal growth restriction appeared in $18.1 \%$ and $16.1 \%$, respectively. Patients with recommended treatment had a good livebirth rate $(85 \%)$, while patients with no treatment showed a poor birth rate $(49.6 \%)$.

Conclusion: In this series, recurrent miscarriage is the most frequent poor outcome. To avoid false-negative diagnoses, all laboratory category subsets were needed. OAPS cases have very good foetal-maternal outcomes when treated. Results suggest that we were able to improve our clinical practice to offer better treatment and outcomes to OAPS patients.

\section{REFERENCES}

[1] Cervera R, Piette JC, Font J, Khamashta MA, Shoenfeld Y, Camps MT, et al. Antiphospholipid syndrome: clinical and immunologic manifestations and patterns of disease expression in a cohort of 1,000 patients. Arthritis Rheumatol 2002;46:1019-1027.

[2] Miyakis S, Lockshin MD, Atsumi T, Branch DW, Brey RL, Cervera R, et al. International consensus statement on an update of the classification criteria for definite antiphospholipid syndrome (APS). J Thromb Haemost 2006:4:295-306.

[3] Esteve-Valverde E, Ferrer-Oliveras R, Alijotas-Reig J. Obstetric antiphospholipid syndrome. Rev Clin Esp 2016;216:135-45.

Acknowledgement: Elmina Lefkou, Gerard Espinosa, Sara Tabacco, Luca Marozio, Pier Luigi Meroni, Tania Melnychuk, V Canti, Vittorio Pengo, Beatrice Chighizola, Maria Gerosa, Elisa Picardo, Elisa Llurba, Arsene Mekinian, Bettina Toth, Katharina Bremme,Ariela Hoxha,

Disclosure of Interests: Enrique Esteve-Valverde: None declared, Jaume Alijotas-Reig: None declared, Raquel Ferrer-Oliveras: None declared, Luis Sáez-Comet: None declared, Cristina Belizna: None declared, Amelia Ruffatti: None declared, Angela Tincani Consultant for: UCB, Pfizer, Abbvie, BMS, Sanofi, Roche, GSK, AlphaSigma, Lillly, Jannsen, Cellgene, Novartis, Sara DeCarolis: None declared, Omar Latino: None declared, Ricard Cervera: None declared

DOI: 10.1136/annrheumdis-2019-eular.2780

\section{$\mathrm{AB} 0166$ \\ ENHANCING THE QUALITY OF CLINICAL DATA THROUGH DATA CURATION IN PRIMARY SJÖGREN'S SYNDROME}

Vasileios Pezoulas ${ }^{1}$, Themis Exarchos ${ }^{1,2}$, Aliki Venetsanopoulou ${ }^{3}$,

Evangelia Zampeli ${ }^{4}$, Saviana Gandolfo ${ }^{5}$, Salvatore De Vita ${ }^{5}$, Foteini N. Skopouli ${ }^{6}$, Athanasios Tzioufas ${ }^{3}$, Dimitrios Fotiadis ${ }^{1} .{ }^{1}$ University of loannina, Dept. of Materials Science and Engineering, Ioannina, Greece; ${ }^{2}$ Ionian University, Dept. of Informatics, Corfu, Greece; ${ }^{3}$ University of Athens, Dept. of Pathophysiology, Athens, Greece; ${ }^{4}$ Institute for Systemic Autoimmune and Neurological Diseases, Athens, Greece; ${ }^{5}$ Udine University, Dept. of Medical and Biological Sciences, Udine, Italy, ${ }^{6}$ Euroclinic Hospital, Dept. of Internal Medicine and Clinical Immunology, Athens, Greece

Background: Primary Sjögren's Syndrome (pSS) is a chronic systemic autoimmune disease that is affecting primarily women near the menopausal age, causing exocrine gland dysfunction, with clinical manifestations varying from dry eye and mouth to multi-systemic disorders [1]. The lack of automated means for data quality improvement in pSS cohorts and the huge time effort needed for manual curation, yield data that are irrelevant and incomplete, introducing undesirable implications in their analysis.

Objectives: To enhance the quality of the clinical data in pSS using automated data curation.

Methods: Anonymized clinical data were recruited from 380 patients with pSS from the University of Athens (UoA) cohort (300 patients, mean age 68.7914.84) and the Harokopio University of Athens (HUA) cohort (80 patients, mean age 59.2913.92). The features consist of SS-related measures (see [2] for details). The curation tool produces 3 files: (i) a quality report, including the metadata, the presence of outliers (using the $z$-score [3]), unknown data types, and missing values, on a feature-basis (data imputation [4] is used to fix features with $<50 \%$ missing values), (ii) the curated dataset, where the inconsistencies are marked using color notations, and (iii) a standardization report, where the features that share common terminology with those from a reference model [5] (i.e., a set of parameters that describe the pSS minimal requirements) are identified using lexical matching [6].

Results: For the UoA cohort, out of 167 features, 80 were classified as "bad", 30 with unknown data type, and 12 were marked for outliers (Fig. 1). An example of an outlier was found for the $\operatorname{lgM}(1370 \mathrm{mg} / \mathrm{dL})$. For the HUA cohort, out of 204 features, 69 were classified as "bad", 5 with unknown data type, and 13 were marked for outliers. The standardization process succesfully matched 82 out of 88 (93.18\%) pSS-related terms for the UoA cohort and 61 out of 69 (88.4\%) terms for the HUA cohort.

Conclusion: Our strategy enhances the quality of the pSS clinical data through data curation and reduces the time effort needed for manual curation by the clinicians. The tool produces re-usable reports that can be used to fix inconsistencies, outliers, missing values, and harmonize pSS clinical data [6].

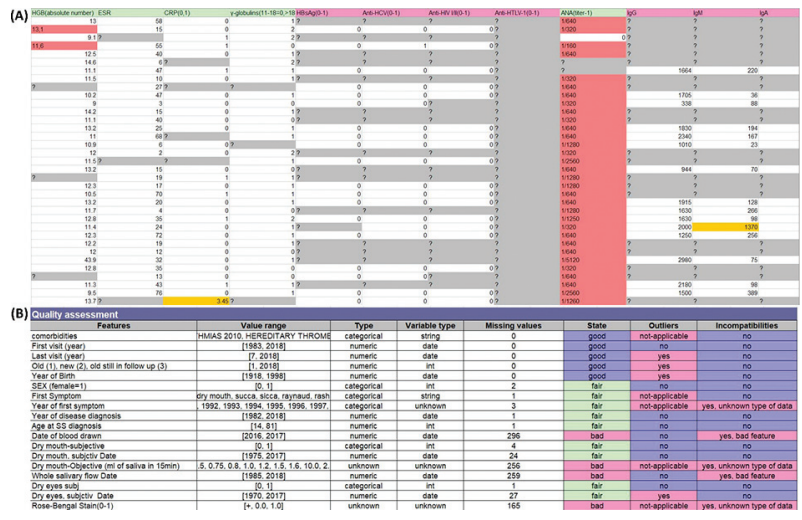

Figure 1. An instance of $(A)$ the curated dataset, and $(B)$ the quality assessment report, for the UoA cohort.

\section{REFERENCES}

[1] C. P. Mavragani, and H. M. Moutsopoulos, "Sjögren syndrome," Can. Med. Assoc. J., 2014;186(15):E579-86.

[2] S. Fragkioudaki, et al., "Predicting the risk for lymphoma development in Sjogren syndrome: an easy tool for clinical use," Medicine, 2016;95(25).

[3] P. J. Rousseeuw, and M. Hubert, "Robust statistics for outlier detection," Wiley Interdiscip. Rev. Data Min. Knowl. Disc., 2011;1(1):73-9.

[4] S. Van Buuren, "Flexible imputation of missing data," Chapman and Hall/ CRC, USA, 2018. 
[5] V. C. Pezoulas, et al., "Towards the establishment of a biomedical ontology for the primary Sjögren's Syndrome," in IEEE Eng. Med. Biol. Soc., 2018;4089-92.

[6] K. Kourou, et al., "Cohort Harmonization and Integrative Analysis from a Biomedical Engineering Perspective," IEEE Rev. Biomed. Eng., 2018.

Acknowledgement: * This project has received funding from the European Union's Horizon 2020 research and innovation programme under grant agreement No 731944 and from the Swiss State Secretariat for Education, Research and Innovation SERI under grant agreement 16.0210.

Disclosure of Interests: Vasileios Pezoulas: None declared, Themis Exarchos: None declared, Aliki Venetsanopoulou: None declared, Evangelia Zampeli Speakers bureau: Roshe, Astrazeneca, Saviana Gandolfo: None declared, Salvatore De Vita Grant/research support from: Roche, Pfizer, Abbvie, Novartis, BMS, MSD, Celgene, Janssen, Consultant for: Roche, Foteini N. Skopouli: None declared, Athanasios Tzioufas Grant/research support from: ABBVIE, PFIZER, AMGEN, NOVARTIS, GSK, Dimitrios Fotiadis: None declared DOI: 10.1136/annrheumdis-2019-eular.4139

\section{AB0167 1 SINGLE CELL RNA EXPRESSION IN LUPUS NEPHRITIS COMPARING AFRICAN-AMERICAN AND CAUCASIAN PATIENTS IDENTIFIES DIFFERENTIAL EXPRESSION OF TYPE I INTERFERON PATHWAY}

Andrea Fava ${ }^{1}$, Yuji Zhang ${ }^{2}$, Nir Hacohen ${ }^{3}$, Arnon Arazi ${ }^{4}$, Celine Berthier ${ }^{5}$, Deepak Rao ${ }^{3,6}$, Michael Brenner ${ }^{3,6}$, David Wofsy ${ }^{7}$, Anne Davidson $^{8}$, Matthias Kretzler ${ }^{9}$, David Hildeman ${ }^{10}$, E. Steve Woodle ${ }^{10}$, Betty Diamond ${ }^{8}$, Michelle A. Petri ${ }^{1}$, on behalf of Accelerating Medicine Partnership. ${ }^{1}$ Johns Hopkins University, Baltimore, United States of America; ${ }^{2}$ University of Maryland, Biostatistics, Baltimore, United States of America; ${ }^{3}$ Harvard Medical School, Boston, United States of America; ${ }^{4}$ Broad Institute, Cambridge, United States of America; ${ }^{5}$ University of Michigan, Nephrology, Ann Arbor, United States of America; ${ }^{6}$ Brigham and Women's Hospital, Boston, United States of America; ${ }^{7}$ UCSF Medical Center, San Francisco, United States of America; ${ }^{8}$ The Feinstein Institute for Medical Research, Manhasset, United States of America; ${ }^{9}$ University of Michigan, Ann Arbor, United States of America; ${ }^{10}$ University of Cincinnati, Cincinnati, United States of America

Background: African-American ethnicity is associated with a 3-fold higher risk of developing systemic lupus erythematosus (SLE). In addition, there is an increased risk of lupus nephritis (2-fold), high-risk histological features, and resistance to treatment. This may account for the increased mortality rate compared to Caucasian patients, especially in women.

Objectives: In Phase One of the Accelerating Medicines Partnership (AMP) study, we used single-cell RNA sequencing on kidney biopsies from patients with active lupus nephritis to identify pathways that were differentially expressed in African-American patients.

Methods: Single cell RNA sequencing was performed on renal biopsies obtained for clinical purpose for active nephritis using CEL-Seq2. Cell clusters with similar expression profile were identified using t-distributed stochastic neighbor embedding (t-SNE). First, the relative abundance of a cluster in AAs compared to Caucasian was determined using a logistic mixed model. Second, the differential expression profile was determined for each cell cluster and we applied Ingenuity Pathway Analysis (IPA) (QIAGEN Bioinformatics) to identify pathways of interest. Results: Samples from 13 AA and 7 Caucasian patients were obtained. Of the 3097 sequenced cell libraries, we used 2354 which passed our quality filter for a total of 30155 unique molecular identifiers. We identified 16 cell clusters including CD4, CD8, B and plasma cells, NK, myeloid cells, and tubular cells. We identified 2 cell clusters unique to African-American patients, a $T$ and a B cell population with high expression of interferon inducible genes. We also identified that same cell populations may have differential gene expression profiles across ethnicity. For example, CD4 T cells in African-Americans have a higher expression of type 1 and type 2 interferon pathways. In contrast, myeloid cells have several upregulated pathways in Caucasians, including ERK/MAPK signaling.

Conclusion: African-American lupus nephritis patients may have a stronger interferon pathway activation in infiltrating immune cells. Several other pathways, including ERK/MAPK, are differentially expressed in infiltrating cells based on ethnicity. These results suggest that ethnicity might predict a response to both current and upcoming treatments, paving the way for a more personalized approach to treatment in lupus nephritis. Further work in Phase 2 of AMP will confirm and extend these findings.

Disclosure of Interests: Andrea Fava: None declared, Yuji Zhang: None declared, Nir Hacohen: None declared, Arnon Arazi: None declared, Celine Berthier: None declared, Deepak Rao: None declared, Michael Brenner Grant/research support from: Roche: sponsored research agreement on stromal cells (but has nothing to do with checkpoint related disease), Consultant for: GSK: consultant. (I am part of their immunology network, a group of about 8 immunologists who advise them regularly and broadly in the areas of inflammation and infection)., David Wofsy Consultant for: GlaxoSmithKline - Member, data safety monitoring board

Novartis - Member, data safety monitoring board

Celgene - member, scientific advisory board, Anne Davidson: None declared, Matthias Kretzler: None declared, David Hildeman: None declared, E. Steve Woodle: None declared, Betty Diamond: None declared, Michelle A Petri Share holder of: Pfizer, Merck, Grant/research support from: AstraZeneca, Exagen, Consultant for: Eli Lilly, GSK, Merck EMD Serono, Janssen, Amgen, Novartis,

Quintiles, Exagen, Inova Diagnostics, AstraZeneca, Blackrock,

Glenmark, UCB, and the Annenberg Center for Health Sciences DOI: 10.1136/annrheumdis-2019-eular.5673

\section{$\mathrm{AB} 0168$ \\ 1,25 DIHYDROXYVITAMIN D IMPROVEMENTS ARTHRITIS BUT DOES NOT ALLEVIATE RENAL DAMAGE IN PRISTANE-INDUCED LUPUS MODEL}

Eduarda Freitas $^{1,2}$, Thaís Karnopp ${ }^{1,2}$, Jordana Miranda de Souza Silva ${ }^{1,2}$, Rafaela Cavalheiro Do Espírito Santo ${ }^{1,2}$, Thales Hein Da Rosa ${ }^{1,2}$, Mayara Souza de Oliveira ${ }^{1}$, Fabiany Gonçalves ${ }^{2}$, Francine Hehn de Oliveira ${ }^{3}$, Pedro Guilherme Schaefer ${ }^{3}$, Odirlei Monticielo ${ }^{1,2}$. ${ }^{1}$ Universidade Federal do Rio Grande do Sul, Porto Alegre, Brazil; ${ }^{2}$ Laboratório de Doenças Autoimunes, Porto Alegre, Brazil; ${ }^{3}$ Hospital de Clínicas de Porto Alegre, Porto Alegre, Brazil

Background: SLE is an autoimmune disease, characterized by the production of autoantibodies and tissue damage. The role of vitamin D (VD) in SLE has been increasingly explored. VD is a steroid hormone notably involved in the regulation of calcium and bone homeostasis, and its deficiency has been implicated in the development of SLE and other connective tissue diseases $(2,3)$. The study of different murine models has provided a better understanding of these autoimmune phenomena. Pristane-induced lupus (PIL) represents a suitable model to study factors that could influence the induction and/or progression of SLE, including genetic factors.

Objectives: To evaluate the development and evolution of SLE after 1,25(OH)2D supplementation in the PIL model.

Methods: Female BALB/c mice divided into 3 groups: $C O, P I L$ and PIL+VD. Lupus was induced in PIL and VD groups using pristane. VD group received a subcutaneous injection of calcitriol [2ug/kg] in PBS-Tween 20 buffer every second day during 180 days. Body weight, arthritis clinical score, edema and articula nociception was measured. On day 150 after pristane induction, the animals were placed in individual metabolic cages for urine collection for a period of $12 \mathrm{~h}$. Protein levels on urine were analyzed using urine test strips. At the end of the experimental period, serum, tibiotarsal joint and kidneys was collected. Hind paws were collected to confirm the development of arthritis by histological analysis with HE staining. The glomerular cellularity was quantified by counting the total cell nuclei per glomerulus on HE slides. Immune complex deposition ( $\lg \mathrm{G}$ and $\operatorname{lgM}$ ) in kidney was examined by direct immunofluorescence. IL-2, IL-4, IL-6, IFN- $\gamma$ and TNF- $\alpha$ were measured by Luminex technology in serum. Data was analyzed with ANOVA Two-Way followed by Bonferroni and independent sample t-test. $p<0.05$ was considered significant. All data are represented as mean \pm SD.

Results: PIL group showed arthritis and kidney injury, characterized by increased proteinuria, glomerular mesangial expansion and inflammation. Moreover, PIL model showed increased levels of IL-6, TNF- $\alpha$ and IFN- $\gamma$ in serum. VD treatment reduced arthritis incidence compared $\mathrm{PIL}(42 \mathrm{vs} 85 \% ; \mathrm{p}<0.01)$ at the end of the experimental period. The arthritis clinical score $(1.00 \pm 1.15 v s 2.85 \pm 1.34 ; p<0.001)$ and the hind paws edema $(0.20 \pm 0.03 v s 0.24 \pm 0.05 \mathrm{~mL} ; \mathrm{p}<0.05)$ in the VD group were also attenuated in relation to the PIL at day 180 after induction. VD was able to reduce synovial hyperplasia $(0(0,1) v s 2(2,3) ; \mathrm{p}<0.05)$, erosion in bone $(0(0,1)$ vs2(2,2);p<0.05) and cartilage $(1(0,2) v s 3(2,3) ; p<0.05)$ when compared to the PIL group. Treatment with VD was not able to reduce proteinuria levels $(44.28 \mathrm{vs} 47.14$ $\mathrm{mg} / \mathrm{dL})$, decrease mesangial hypercellularity (31.48 $\pm 2.6 \mathrm{vs} 33.12 \pm 3.4$ nucleo/glo meruli) or IgG (17.88 $\pm 6.0 v s 24.04 \pm 6.2)$ and $\lg M(12.62 \pm 6.9 v s 15.57 \pm 3.7)$ deposition in the kidney. VD supplementation did not alter IL-6, TNF- $\alpha, \mathrm{IL}-2$ and IL-4 cytokine levels, but reduce IFN- $y$ levels $(p<0.01)$

Conclusion: VD improves arthritis but does not influence renal injury despite reducing IFN-y levels. These results support that the role of VD may be different depending on acting site, what could explain different responses according clinical phenotype. Therefore, further investigations of VD are needed to explore the supplement dosage, timing, and the molecular basis in SLE.

\section{REFERENCES}

[1] Pons-Estel GJ, et al. Seminars in Arthritis and Rheumatism. 2010. 2 Kamen DL, et al. Autoimmun Rev 2006; 5: 114-117. 3 Orbach H, et al. Ann N Y Acad Sci 2007; 1109: 385-400.

Acknowledgement: FIPE/HCPA, CAPES, CNPq Universal

Disclosure of Interests: None declared

DOI: 10.1136/annrheumdis-2019-eular.6328 